\title{
A Few Problems on Monodromy and Discriminants
}

\author{
V. A. Vassiliev 1
}

Received: 19 February 2015 / Accepted: 31 March 2015 / Published online: 16 April 2015

(C) Institute for Mathematical Sciences (IMS), Stony Brook University, NY 2015

\begin{abstract}
The article contains several problems concerning local monodromy groups of singularities, Lyashko-Looijenga maps, integral geometry, and topology of spaces of real algebraic manifolds.
\end{abstract}

Keywords Morsification · Lyashko-Looijenga map · Covering number · Versal deformation $\cdot$ Real algebraic geometry

\section{Explicit Obstructions to the Lyashko-Looijenga Covering (and its Real Analogs) for Non-Simple Singularities}

The so-called Lyashko-Looijenga covering (see Looijenga 1974; Vassiliev 2002a) is a strong tool for constructing (or proving the existence of) the perturbations of simple singularities with prescribed topological properties, such as singularity types of different critical points, or intersection matrices of vanishing cycles: see, e.g., Lyashko (1976). The real version of this tool allows one to construct and enumerate all topologically different Morsifications of real simple singularities: see Looijenga (1978), Chislenko (1988), Vassiliev (2002b).

Many of these options are preserved for non-simple singularities: see Vassiliev (2002a,b). In particular, this method was used to predict the existence of many Morsifications with prescribed properties and to describe their expected topological

Supported by Program "Leading scientific schools", Grant No. NSh-5138.2014.1 and RFBR Grant 13-01-00383.

$凶 \quad$ V. A. Vassiliev vva@mi.ras.ru

1 Steklov Mathematical Institute and National Research University — Higher School of Economics, Moscow, Russia 
characteristics, making it easy to give an explicit construction of these Morsifications. However, in this case this method is rather experimental or heuristic, without clear guaranties that all perturbations found by it actually exist. Therefore, it is important to track down the restrictions of this method. Here are several explicit problems.

\subsection{Complex Version}

Let $f:\left(\mathbb{C}^{n}, 0\right) \rightarrow\left(\mathbb{C}^{1}, 0\right)$ be an isolated holomorphic function singularity, $\mu$ be its Milnor number, $F(x, \lambda):\left(\mathbb{C}^{n} \times \mathbb{C}^{\mu}, 0\right) \rightarrow\left(\mathbb{C}^{1}, 0\right)$ be the miniversal deformation of $f$, and $\Sigma \subset \mathbb{C}^{\mu}$ be the complete bifurcation set of functions of this deformation, i.e. the set of values of the parameter $\lambda \in \mathbb{C}^{\mu}$ such that the corresponding function $f_{\lambda}=F(\cdot, \lambda)$ has less than $\mu$ different critical values at critical points close to the origin. The Lyashko-Looijenga map sends any point $\lambda$ from a small neighborhood $B_{\varepsilon}$ of the origin in $\mathbb{C}^{\mu}$ to the unordered collection of critical values of the function $f_{\lambda}$ at points close to $0 \in \mathbb{C}^{n}$ (or, which is equivalent but sometimes more convenient, to the set of values of basic symmetric polynomials of these critical values). If the singularity of $f$ is simple, then the restriction of this map to $B_{\varepsilon} \backslash \Sigma$ is a local covering over the configuration space $B(D, \mu)$ of all subsets of cardinality $\mu$ in a very small (even with respect to $\varepsilon$ ) neighborhood $D$ of the origin in $\mathbb{C}$ : see Looijenga (1974). In particular, any element $\alpha \in \pi_{1}(B(D, \mu))$ can be realized by a loop which can be lifted to a path in $B_{\varepsilon} \backslash \Sigma$, covering this loop.

For non-simple singularities this is no longer the case. As previously, the LyashkoLooijenga map is submersive (and hence locally bijective) everywhere in $B_{\varepsilon} \backslash \Sigma$ (this follows from the very notion of miniversality). However, a sufficiently complicated path in $B(D, \mu)$, lifted into $\mathbb{C}^{\mu} \backslash \Sigma$ in accordance with this local bijectivity, can run out from the neighborhood of the origin in $\mathbb{C}^{\mu}$. This is related to the fact that the Lyashko-Looijenga map for non-simple singularities is not proper: the preimage of the collection $(0, \ldots, 0)$ is the entire (positive-dimensional) $\mu=$ const stratum. $^{1}$

Problem 1A Present explicit obstructions to the Lyashko-Looijenga covering in terms of braid groups. Which braids cannot be lifted to the space $\mathbb{C}^{\mu} \backslash \Sigma$ ?

Given a configuration of $\mu$ different points $z_{1}, \ldots, z_{\mu}$ in $D \backslash 0$ and a system of nonintersecting paths connecting them with 0 , any perturbation $f_{\lambda}$ of $f$, which has these critical values, defines a Dynkin diagram: see Arnold et al. (1985), vol. 2. Any braid $l \in \pi_{1}(B(D, \mu))$ transforms this Dynkin diagram into another one in accordance with the Picard-Lefschetz formulas: see Arnold et al. (1985) or Vassiliev (2002a). If our braid $l$ can be lifted to a curve in $B_{\varepsilon} \backslash \Sigma$ starting at the point $\lambda$ and covering this braid via the Lyashko-Looijenga map, then the resulting Dynkin diagram is nothing but the Dynkin diagram of the function $f_{\lambda^{\prime}}$ corresponding to the endpoint of this lifted curve and defined by the same system of paths connecting the critical values with 0 .

However, for complicated singularities, the number of Dynkin graphs, which can be obtained by the formal Picard-Lefschetz moves, is infinite, while the number of

\footnotetext{
1 A weaker substitute for the Lyashko-Looijenga covering theorem holds in the case of parabolic singularities, if one writes the versal deformation in the canonical monomial form and allows large travellings in the space $\mathbb{C}^{\mu}$ : see Jaworski (1986). For more complicated singularities the situation is even worse.
} 
preimages of any non-discriminant configuration under the Lyashko-Looijenga map is bounded.

Problem 1B Let a non-simple singularity $f$ be given and the Dynkin diagram of it be defined by an easily distinguished system of paths connecting 0 with critical points of $f_{\lambda}$. Which Dynkin graphs can be obtained from it by a sequence of formal Picard-Lefschetz moves defined by a braid, but cannot appear as Dynkin diagrams of Morsifications $f_{\lambda^{\prime}}$ with the same critical values defined by the same system of paths?

Furthermore, for simple singularities, all partial collisions of $\mu$ critical values can be realized, because the Lyashko-Looijenga map is proper. This reduces the problem of enumeration of possible decompositions of the initial critical point to a problem formulated in terms of Dynkin diagrams and Picard-Lefschetz operators only: see Lyashko (1976). Again, for non-simple singularities, it is not the case. For example, any non-simple singularity admits a system of paths connecting 0 with critical values, such that there are two vanishing cycles with the intersection number equal \pm 2 . Then we surely cannot lift to $B_{\varepsilon}$ the collision of these two critical values along these paths while keeping the remaining critical values unmoved. Namely, the attempt to move these critical values towards one another by means of the Lyashko-Looijenga submersion will throw the parameter $\lambda$ out of any neighborhood of the origin in $\mathbb{C}^{\mu}$.

Problem 1C Are there more refined restrictions to the collision of critical values? Is it true that for any two vanishing cycles, whose intersection number is equal to \pm 1 or 0 , we can lift the collision of the corresponding critical values to $B_{\varepsilon}$ via the Lyashko-Looijenga submersion?

In the previous consideration, the existence of two vanishing cycles with the intersection number \pm 2 ensures the non-properness of the Lyashko-Looijenga map, and hence the fact that the $\mu=$ const stratum of the singularity is positive-dimensional.

Problem 1D Give more general lower bounds of the dimension of $\mu=$ const strata in terms of intersection forms of vanishing cycles.

That is, if we can indicate many independent prohibited collisions of critical values, then probably the attempt to perform these collisions by brute force will throw the corresponding Morsifications out of the neighborhood of the origin in $\mathbb{C}^{\mu}$ in independent directions (all of which approach the $\mu=$ const stratum).

\subsection{Real Version}

The real versions of these problems are important for the construction of real decompositions and enumeration of topologically distinct Morsifications of real singularities: see Chislenko (1988), Vassiliev (2002b). Namely, let $f:\left(\mathbb{C}^{n}, \mathbb{R}^{n}, 0\right) \rightarrow(\mathbb{C}, \mathbb{R}, 0)$ be a real function singularity, and $F:\left(\mathbb{C}^{n} \times \mathbb{C}^{k}, \mathbb{R}^{n} \times \mathbb{R}^{k}, 0\right) \rightarrow(\mathbb{C}, \mathbb{R}, 0)$ be its real deformation. The space $\mathbb{R}^{k}$ of real parameters is separated into several chambers by the real total discriminant (consisting of all non-Morse functions and functions with critical value 0). We can go from any chamber to any other one by a generic path 
in $\mathbb{R}^{k}$ passing only finitely many times the discriminant at its non-singular points. Any such passage changes the topological type of the function $f_{\lambda}$ in some predictable way. Moreover, if our singularity $f$ is simple and the deformation $F$ is versal, then all standard changes satisfying some natural restrictions can indeed be performed. In particular, if $f_{\lambda}$ has two neighboring real critical values, then we can collide them and get two critical points on the same level (if the intersection number of corresponding vanishing cycles is 0 ) or a critical point of type $A_{2}$ (if this number is \pm 1 ). In the latter case, these two critical values (and the corresponding critical points) go to the imaginary domain after this passage.

For non-simple singularities, we can perform all the same formal surgeries over the collections of critical values (supplied with the intersection matrix and some additional set of topological invariants of a real Morsification), and combine these formal surgeries in arbitrary sequences.

Problem 1E What are the obstructions to the realization of these chains of formal changes by paths in the parameter space $\mathbb{R}^{k}$ ?

An algorithm enumerating all such chains of surgeries was realized in Vassiliev (2002b). This algorithm was executed for all singularities of corank 2 and $\mu \leq 11$, and this execution never met a formal surgery which could not be realized by a surgery of functions in the versal deformation.

Can this experimental fact be raised to the theorem level?

\subsection{Prediction of the Indices of Newborn Critical Points at a Morse Surgery}

Consider a one-parameter family of real analytic functions (or just polynomials) $f_{\tau}:\left(\mathbb{C}^{n}, \mathbb{R}^{n}\right) \rightarrow(\mathbb{C}, \mathbb{R}), \tau \in(-\varepsilon, \varepsilon)$ realizing a Morse birth surgery: the functions $f_{\tau}, \tau<0$, have two complex conjugate critical points which collide in a point of type $A_{2}$ when $\tau$ approaches 0 , and after that reappear as two real Morse critical points of some two neighboring Morse indices.

Problem 1F Is there any convenient topological characteristic of the function $f_{-\varepsilon}$ which allows to predict these indices?

The parities of these indices can indeed be predicted. Namely, consider the complex level manifold $V_{a}=f_{-\varepsilon}^{-1}(a)$, where $a$ is a real non-critical value between complex conjugate critical values of $f_{-\varepsilon}$ which are going to collide, and vanishing cycles in this manifold defined by segments connecting $a$ with these critical values. The intersection number of these cycles is equal to \pm 1 depending on the choice of their orientations. Let us choose these orientations in such a way that the complex conjugation in $V_{a}$ takes one of them into the other one. Then the sign of their intersection number is well-defined and allows us to guess the parities of the indices of newborn critical points: see Vassiliev (2002a, b). But how can we predict the integer indices? 


\section{Covering Number (Genus) of Maps Which are Not Fiber Bundles}

Given a surjective map of topological spaces, $p: X \rightarrow Y$, the covering number of $p$ is the minimal number of open sets covering $Y$ in such a way that there is a continuous cross-section of $p$ over any of these sets. This definition was given by Smale (1987) in connection with the problems of complexity theory. In the particular case of fiber bundles, this notion was earlier introduced and deeply studied by Schwarz (1966) under the name of the genus of a fiber bundle. However, in the complexity theory of equations over real numbers, the case of maps with varying fibers becomes essential. Here is one of the first examples. Consider the 6-dimensional real space of pairs of polynomials $\left(f_{a}, g_{b}\right): \mathbb{R}^{2} \rightarrow \mathbb{R}^{2}$, where $f_{a}(x, y)=x^{2}-y^{2}+a(x, y), g_{b}=x y+b(x, y)$, and $a(x, y)$ and $b(x, y)$ are arbitrary polynomials of degree $\leq 1$. Obviously, the system $\left\{f_{a}=0, g_{b}=0\right\}$ always has 2 or 4 solutions in $\mathbb{R}^{2}$ (counted with multiplicity).

Problem 2A What is the minimal number of open sets $U_{i}$ covering $\mathbb{R}^{6}$ such that for any $U_{i}$ there is a continuous map $\varphi_{i}: U_{i} \rightarrow \mathbb{R}^{2}$ sending any pair $(a, b) \in U_{i}$ into some solution of the system $\left\{f_{a}=0, g_{b}=0\right\}$ ?

In previous terms, this is a question about the covering number of the projection map $X \rightarrow Y$, where $Y=\mathbb{R}^{6}$ is the space of parameters $(a, b)$, and $X \subset \mathbb{R}^{6} \times \mathbb{R}^{2}$ is the space of pairs $((a, b),(x, y))$ such that $(x, y) \in \mathbb{R}^{2}$ is a root of the system $\left\{f_{a}=0, g_{b}=0\right\}$.

The number in question is not less than 2 (indeed, we can emulate the complex equation $z^{2}=A$ inside our system, and the covering number of this equation depending on the complex parameter $A$ is equal to 2). But is this estimate sharp?

Problem 2B The same questions concerning the approximate solutions. That is, for any $i$ and any $(a, b) \in U_{i}$, the value $\varphi_{i}(a, b)$ should be not necessarily a root of the system $\left\{f_{a}=0, g_{b}=0\right\}$, but just a point in the $\varepsilon$-neighborhood of such a root for some fixed positive $\varepsilon$.

These problems have obvious generalizations to polynomial systems of higher degrees and different numbers of variables. They can be non-trivial even for polynomials in one real variable: see Vassiliev (2014).

\section{$3 K(\pi, 1)-P r o b l e m$ for the Complement of the Essential Ramification Set of a General Real Polynomial in One Variable}

Consider the space $\mathbb{R}^{d}$ of all real polynomials

$$
f_{a}(x)=x^{d}+a_{1} x^{d-1}+\cdots+a_{d-1} x+a_{d}, \quad a_{j} \in \mathbb{R} .
$$

The essential ramification set in the space $\mathbb{R}^{d}$ is the union of all values $a=\left(a_{1}, \ldots, a_{d}\right)$ such that the corresponding polynomial $f_{a}$ has either a real triple root, or a pair of complex conjugate imaginary double roots: see Vassiliev (2011). Obviously, this set is a subvariety of codimension 2 in $\mathbb{R}^{d}$.

Problem 3 Is the complement of the essential ramification set in $\mathbb{R}^{d}$ a $K(\pi, 1)$-space? 


\section{Odd-Dimensional Newton's Lemma on Integrable Ovals and Geometry of Hypersurfaces}

This is actually the "odd-dimensional part" of Arnold's problem 1987-14 from Arnold (2004) (repeated as problem 1990-27). I describe below its reduction to a problem in algebraic geometry.

Any compact domain in $\mathbb{R}^{n}$ defines a two-valued function on the space of affine hyperplanes: the volumes of two parts into which the hyperplane cuts the domain. If $n$ is odd and the domain is bounded by an ellipsoid, then this function is algebraic (by a generalization of Archimedes' theorem on sphere sections).

Arnold's problem (see Arnold 2004). Do there exist smooth hypersurfaces in $\mathbb{R}^{n}$ (other than the quadrics in odd-dimensional spaces), for which the volume of the segment cut by any hyperplane from the body bounded by them is an algebraic function of the hyperplane?

Many obstructions to the algebraicity of the volume function follow from the Picard-Lefschetz, theory, studying the ramification of integral functions: see Vassiliev (2002a), Arnold and Vassiliev (1989). These obstructions are quite different in the cases of even or odd $n$, because the homology intersection forms, which are a major part of the Picard-Lefschetz formulas, behave very differently depending on the parity of $n$. In particular, the "even-dimensional" obstructions are sufficient to prove that the volume function of a compact domain with $C^{\infty}$-smooth boundary in $\mathbb{R}^{2 k}$ is never algebraic: see Vassiliev (2015). Here are two similar obstructions specific for the case of odd $n$.

Definition A non-singular point of a complex algebraic hypersurface is called parabolic, if the second fundamental form of the hypersurface (or, equivalently, the Hessian matrix of its equation) is degenerate at this point. A parabolic point $x$ is degenerate, if the tangent hyperplane to our hypersurface at $x$ is tangent to it at entire variety of positive dimension containing the point $x$.

Proposition (see Vassiliev 2002a). If $n$ is odd and the volume function defined by $a$ bounded domain with smooth boundary in $\mathbb{R}^{n}$ is algebraic, then the complexification of this boundary cannot have non-degenerate parabolic points in $\mathbb{C}^{n}$.

Smooth algebraic projective hypersurfaces of degree $\geq 3$ always have parabolic points (and moreover, by a theorem of F. Zak, they have only non-degenerate parabolic points). Unfortunately, this is not sufficient to give a negative answer to the above Arnold problem, because

(a) the complexification of a smooth real hypersurface can have singular points in the complex domain, and non-smooth hypersurfaces of arbitrarily high degrees can have no parabolic points: for instance, this is the case for hypersurfaces projective dual to smooth ones;

(b) the previous proposition does not prohibit parabolic points in the plane at infinity $\mathbb{C} \mathbb{P}^{n} \backslash \mathbb{C}^{n}$.

However, the standard singular points, which can occur instead of parabolic points, the generic cuspidal edges, also prevent the algebraicity of the corresponding volume function: see Vassiliev (2002a), §III.6. 
Problem 4 Are these geometric obstructions sufficient to solve the above problem?

In other words, is it true that the complexification of the smooth algebraic boundary of degree $\geq 3$ of a compact domain in $\mathbb{R}^{n}$ has always a point of one of these two obstructing types? If not, probably we can complete this list by some other singularity types, which also obstruct the algebraicity, so that singular points of at least one of these types will be unavoidable on any such hypersurface.

\section{Greedy Simplifications of Real Algebraic Manifolds}

Given natural numbers $d$ and $N$, consider the space $P(d ; N)$ of all smooth algebraic hypersurfaces of degree $d$ in $\mathbb{R}^{N}$. The trivial elements of this space are the empty manifolds if $d$ is even, and the surfaces isotopic to $\mathbb{R}^{N-1}$ if $d$ is odd. Consider also some natural measure of topological complexity of such hypersurfaces, which takes the smallest value on trivial objects only: for example, the sum of numbers of generators of homology groups, or the lowest number of critical points of Morse functions.

Problem 5A Is it true that any hypersurface from the space $P(d ; N)$ can be connected with a trivial one by a generic path in this space in such a way that it experiences only Morse surgeries, which decrease this complexity measure?

In other words, does our space contain non-trivial varieties with the following property: any surgery of this variety decreases (or leaves unchanged) this complexity measure?

This problem can be extended to algebraic submanifolds defined by systems of polynomials; however, the measure of topological complexity in this case should take into account the possible "knottedness" in $\mathbb{R}^{N}$.

Problem 5B A version of the previous problem, in which the complexity measure is not purely topological: namely, it is the lowest number of critical points of Morse functions defined by restrictions of linear functions $\mathbb{R}^{N} \rightarrow \mathbb{R}$ to our varieties. (Correspondingly, the surgeries of the variety affecting this measure should be not only those of topological nature but also include bifurcations of the dual variety).

If the answer to the previous questions is negative, then we obtain functions that associate with any value $T$ of topological complexity the lowest number $F$ such that any surface of complexity $T$ can be connected with a trivial one by a generic path in the space $P(d ; N)$ for which the complexities of all intermediate hypersurfaces do not exceed $F$.

Problem 5C Give an upper bound for the function $T \mapsto F$.

It is appropriate to mention here a related problem due to V.A. Rokhlin.

Problem 5D Do non-singular real plane projective curves of an odd degree consisting of a single connected component form a connected set (i.e., are they rigid isotopic)?

The first degree for which the answer is unknown is 7 . 


\section{A Local Version of Problems of Section 5}

Let $f:\left(\mathbb{R}^{n}, 0\right) \rightarrow(\mathbb{R}, 0)$ be a function germ with $d f(0)=0$ and the Milnor number $\mu(f)$ finite. Let $\rho(f)$ be the smallest number of real critical points of real Morsifications of $f$.

Problem 6A Is it true that any real Morsification of $f$ can be connected with one of complexity $\rho(f)$ by a generic path in the base of a versal deformation, in such a way that all Morse surgeries $\left[A_{2}\right]$ in this path only decrease the number of real critical points?

Problem 6B What can be said about the number $\rho(f)$ ?

Two obvious lower estimates of this number are (a) the index of $\operatorname{grad} f$ at 0 , and (b) the Smale number of the relative homology group

$$
H_{*}\left(f^{-1}((-\infty, \varepsilon]), f^{-1}((-\infty,-\varepsilon])\right)
$$

(i.e. the rank of the free part of this group plus twice the minimal number of generators of its torsion). Of course, the first estimate does not exceed the second one, but can they be different? Do they coincide at least for functions of corank 2?

Can the group (1) have non-trivial torsion? Is the estimate (b) of the number $\rho(f)$ sharp?

Problem 6C Is it true that any component of the complement of the discriminant variety of a versal deformation contains a Morsification, whose all $\mu(f)$ critical points are real?

This is true for all simple singularities: see Vassiliev (2002a).

\section{Radius of Convergence for Multidimensional Newton's Method}

Consider a polynomial $\mathbb{C}^{1} \rightarrow \mathbb{C}^{1}$ of degree $n$ and some simple root $z_{0}$. Let $d$ be the minimal distance from this root to all other roots of this polynomial. According to Reshetnyak (1962), the $\frac{d}{2 n-1}$-neighborhood of $z_{0}$ belongs to its domain of convergence; that is, Newton's method starting from any point of this neighborhood converges to $z_{0}$. The estimate $\frac{d}{2 n-1}$ cannot be improved as a function of $d$ and $n$ not depending of the polynomial.

Problem 7 Give a similar universal estimate of the radius of convergence for multidimensional Newton's method of Shub and Smale (1993).

\section{References}

Arnold, V.I., Varchenko, A.N., Gusein-Zade, S.M.: Singularities of Differentiable Maps, vol. 1, 2. Birkhäuser, Basel (1985)

Arnold, V.I.: Arnold's Problems. Springer, New York, PHASIS, Moscow (2004) 
Arnold, V.I., Vassiliev, V.A.: Newton's Principia read 300 years later. Notices AMS 36(9), 1148-1154 (1989)

Chislenko, Yu.S.: Decompositions of simple singularities of real functions. Funct. Anal. Appl. 22(4), 297310 (1988)

Jaworski, P.: Distribution of critical values of miniversal deformations of parabolic singularities. Invent. Math. 86, 19-33 (1986)

Looijenga, E.J.H.: The complement of the bifurcation variety of a simple singularity. Invent. Math. 23(2), 105-116 (1974)

Looijenga, E.J.H.: The discriminant of a real simple singularity. Compos. Math. 37(1), 51-62 (1978)

Lyashko, O.V.: Decomposition of simple singularities of functions. Funct. Anal. Appl. 10(2), 122-128 (1976)

Milnor, J.: Lectures on the $h$-Cobordism Theorem. Princeton University Press, Princeton (1965)

Reshetnyak, Yu.G.: A contribution to the problem of calculating the complex roots of a polynomial by Newton's method. USSR Comput. Math. Math. Phys. 1(4), 1274-1276 (1962)

Schwarz, A.S.: The genus of a fiber space. Trans. Mosc. Math. Soc. 10, 217-272 (Engl. Transl. AMS Transl. 55(1966), 49-140 (1961))

Shub, M., Smale, S.: Complexity of Bezout's theorem 1: geometric aspects. J. AMS 6:2, 459-501 (1993)

Smale, S.: On the structure of manifolds. Am. J. Math. 84, 387-399 (1962)

Smale, S.: On the topology of algorithms. J. Complex. 3(2), 81-89 (1987)

Vassiliev, V.A.: Applied Picard-Lefschetz Theory. American Mathematical Society, Providence (2002a)

Vassiliev, V.A.: A FORTRAN program counting for Morsifications of real function singularities. http:// www.pdmi.ras.ru/arnsem/papers (2002b)

Vassiliev, V.A.: On topological invariants of real algebraic functions. Funct. Anal. Appl. 45(3), 163-172 (2011)

Vassiliev, V.A.: Topology in Arnold's work. In: Khesin, B.A., Tabachnikov, S.L. (eds.) Arnold: Swimming Against the Tide, American Mathematical Society, Providence, pp. 165-171 (2014)

Vassiliev, V.A.: Newton's lemma XXVIII on integrable ovals in higher dimensions and reflection groups. Bull. Lond. Math. Soc. 47(2): 290-300 (2015) 